\title{
Laser polishing of titanium surfaces obtained by additive manufacturing process
}

\author{
Benoit Rosa $^{*}{ }^{1}$ and Jean-Yves Hascoët ${ }^{1}$ \\ ${ }^{1}$ Institut de Recherche en Génie Civil et Mécanique UMR CNRS 6183, Centrale Nantes, Nantes, France \\ *benoit.rosa@ec-nantes.fr
}

\begin{abstract}
Additive Manufacturing (AM) surfaces are composed by different textures and high roughness values which tend to limit its functionalities. Laser polishing process is enabling to smooth surfaces by material melting, change surface texture and decrease surface roughness $\left(S_{a}\right)$. Based on a five axes machine, which consist of milling and Laser Metal Deposition (LMD) processes, the machine is additionally integrating laser polishing process on the same architecture. This paper aims at study laser polishing of laser metal deposition of titanium surfaces. LMD of titanium surfaces are composed by chaotic texture directly induced by the physical phenomenon of the process in use. Laser polishing process (LP) has an impact on the final surface regarding a multi-scale approach. The determined operating parameters and path strategy of laser polishing process decreases surface roughness by $78 \%$ and allow smoothing the initial chaotic texture. A polished surface roughness of $6.01 \mu \mathrm{m}$ was obtained from an initial of $27.6 \mu \mathrm{m}$.
\end{abstract}

\section{Introduction}

Additive Manufacturing (AM) technology allows creating several simple and complex forms of objects, for many different applications. Actually, many researchers and industries applies AM with different classes of materials such as metal [1], sand [2], glass [3], ceramics [4], polymers [5], wood [6], concrete [7], food [8] and bioprinting [9 and 10] for example.

Globally for all these materials, the process is the same. The additive manufacturing process is based on a numerical chain and allows creating simple and complex form layer by layer deposition. The numerical model of the desired object is obtained through CAD (Computer Aided Design) software and is transferred to CAM (Computer Aided Manufacturing) software for creating spatial manufacturing trajectories that will be apply to the machine.

Additive manufacturing of metal is composed by different technologies: Laser Metal deposition (LMD), wire arc additive manufacturing (WAAM), selective laser melting (SLM) and electron beam melting (EBM).

Based on powder projection, LMD allows functional material gradient creation (FGM) [14] and thin structures. However, LMD surfaces can be composed by roughness values that it tends, in some cases, to limit their functionalities.

Surfaces are composed by different textures directly caused by physic phenomenon of process (Figure 1) [15]. First one is the waviness that is corresponding to the superposed layers of the part. Second one is the chaotic texture, which is caused by the powder projection. The surface characteristics allow analysing the LMD surfaces after finishing process and there functionalities.

In order to improve LMD surface roughness, laser polishing process is used. Laser polishing process allows smoothing surfaces by material melting. When the laser scans the surface of the material is melted and with surface tension the material is smoothed. Laser polishing enables considerably reduction in surface roughness of LMD. Indeed, laser polished surfaces must be characterized by a multi scale analysis which highlight roughness and waviness components.

A few studies on laser polishing of titanium are proposed [16-22], but only one focus partially on laser polishing of LMD titanium surfaces [23]. In this study, laser polished LMD surfaces is investigated, but no multiscale analysis is effectuated. Besides, no micro crack analysis is proposed.

This paper aims at study the laser polishing of thin LMD titanium surfaces regarding multi scale and micro cracks analysis. The operating parameters of laser polishing process have been determined previously on thin 316L surface in order to avoid that the surface collapses. Additionally, these operating parameters are applied to Ti6Al4V surface as starting parameters, also in order to avoid the surface to collapse. Next, the feed rate parameter has been increased for the titanium material to improve productivity. Besides, the enhance of feed rate enables to limit heat transfer of the thin structure. 


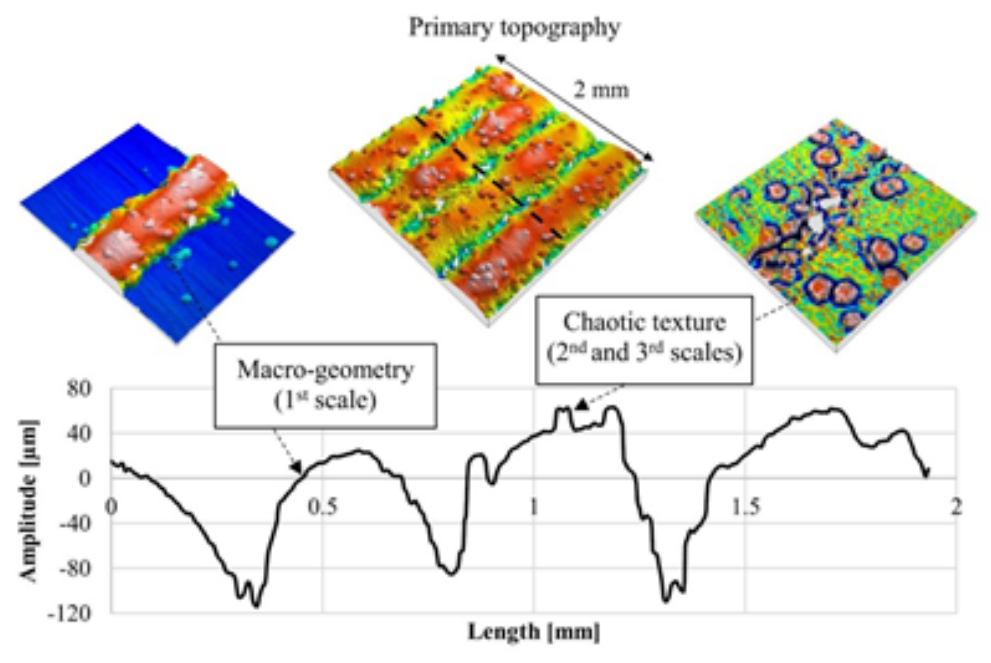

Figure 1: surface topography of LMD surface [15]

A multi scale analysis of the surface is effectuated in order to quantify the impact of the laser polishing process. This study use amplitude, spatial frequency and texture parameters to quantify the surface evolution. Both 316L and Ti6Al4V LMD and laser polished surfaces are compared to highlight the importance to use a multi scale method analysis. Finally, the texture analysis enables to study the impact of the initial LMD topography on the final laser polished topography.

\section{Methodology of investigation}

LMD surfaces are obtained on 5 axis machine (Figure 2a) that is integrated milling, LMD and laser polishing processes. The LMD surface is obtained through a vertical strategy (Figure 2b). This strategy is used to create complex geometries of parts. Argon gas containment cell is used during the process to protect surface from oxidation.

a)

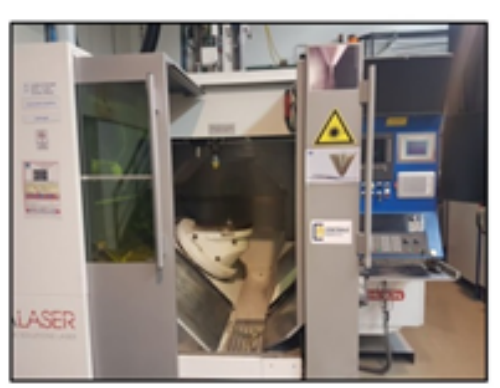

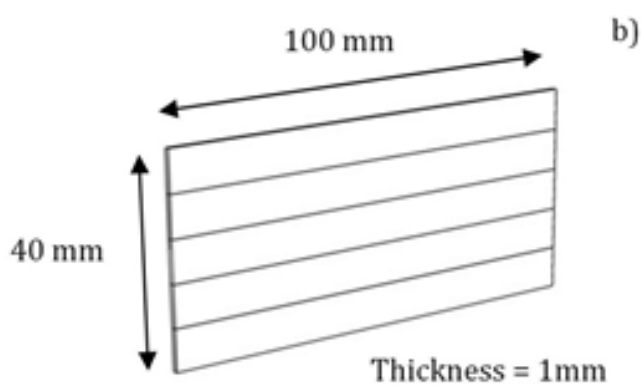

Thickness $=1 \mathrm{~mm}$

b)

Figure 2: hybrid machine a) and dimensions of the LMD titanium surface b)

The used values of laser polishing operating parameters are issued of tests applied to 316L LMD surfaces (Table 1). The tested parameters are the laser power $(P)$ [W], the feed rate $(V f)[\mathrm{mm} / \mathrm{min}]$, the overlap $(O v)[\%]$, that is the distance between two laser tracks along the path direction and the pass number $(P n)$, that is consists in applying $\mathrm{N}$ times the same laser parameters on the same surface according to the same laser path. This strategy enables increase in interaction time between the laser and the surface, without increasing energy density for limit heat transfer for thin structures. The previous works on 316L material have shown that for 4 and 5 passes the surface evolution is limited [23]. For this reason, only 1 and 5 passes are applied to Ti6Al4V surfaces. An overlap of $60 \%$ is selected to avoid surface micro cracks and limit heat transfer. The used laser polishing strategy is a zig zag.

\begin{tabular}{|c|c||}
\hline Operating paramters & values \\
\hline \hline Power $(P)[\mathrm{W}]$ & 210 \\
\hline \hline Feed rate $(V f)[\mathrm{mm} / \mathrm{min}]$ & 3000 \\
\hline \hline Overlap $(O v)[\%]$ & 60 \\
\hline Pass number $(P n)$ & 1 and 5 \\
\hline
\end{tabular}

Table 1: operating parameters of Ti6Al4V laser polishing 
Focus variation microscopy is used to measure LMD and polished surfaces. A 10x and 50x magnifications are used. The lateral resolution is $3.91 \mu \mathrm{m}$ and the vertical is $300 \mathrm{~nm}$ for the 10x and $1.038 \mu \mathrm{m}$ and $20 \mathrm{~nm}$ for the 50x. The measurement device allows measuring large areas and is composed by tilt and rotate automated axes. The surface topography improvement is characterized by surface roughness $\left(S_{a}\right)[\mu \mathrm{m}]$ and surface roughness reduction [\%] (Eq. 1 and 2) [24]. In order to analyse the spatial evolution of the polished topographies, Wsm parameter was used. This parameter helps to characterize the average spacing of profile elements of waviness (Eq.3). Furthermore, Str (texture aspect ratio) parameter and autocorrelation lobe are used to analyse textures isotropy. The autocorrelation image always includes a central peak. If the surface presents the same characteristics in every direction, the central lobe will be approximately circular. If the surface presents a strong privileged orientation, the central lobe will be very stretched out. The Str parameter takes a value between 0 and 1, and is unitless. It can also be expressed as a percentage between $0 \%$ and $100 \%$. An isotropic surface will have Str close to $1(100 \%)$ while a strongly anisotropic surface will have Str close to 0.

$$
\begin{gathered}
S_{a}[\mu m]=\frac{1}{A} \iint_{A}|z(x, y)| d x d y \quad(\mathrm{Eq} .1) \\
S_{a_{\text {reduction }}[\%]}=\frac{s_{a_{\text {initial }}}-S_{a_{\text {polished }}}}{S_{a_{\text {initial }}}} * 100 \quad(\mathrm{Eq} \cdot 2) \\
W s m=\frac{1}{m} \sum_{i=1}^{m} X s i \quad \text { (Eq.3) }
\end{gathered}
$$

\section{Results}

Compared to the $316 \mathrm{~L}$ surfaces, the LMD titanium surfaces are composed by different textures (Figure 3). LMD titanium surfaces are composed by chaotic texture and without regular waviness. The regular waviness is due to the superposition layer of material during additive manufacturing process. As a result, the LMD textures directions are different. The surface roughness of the $316 \mathrm{~L}$ surface is $23.98 \mu \mathrm{m}$ and $27.6 \mu \mathrm{m}$ for the Ti6Al4V LMD surface respectively. The surface roughness values have been calculated with a $8000 \mu \mathrm{m}$ cut off filter $(\lambda \mathrm{c})$.
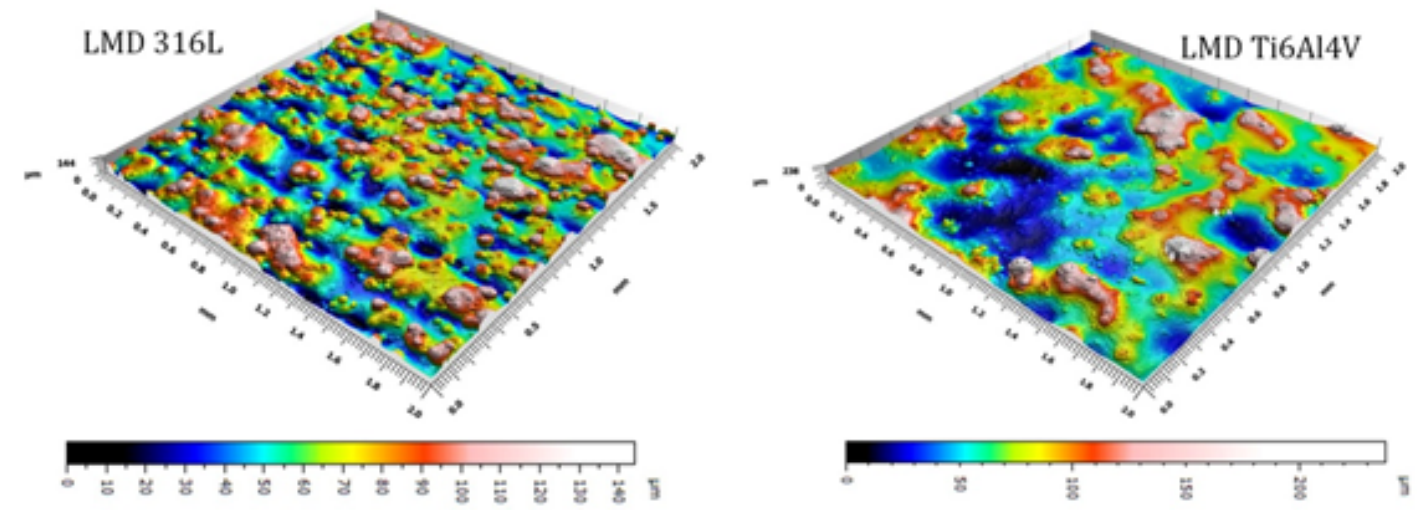

Figure 3: comparison between 316L and Ti6AI4V LMD surfaces

Laser polishing process allows to smooth LMD surface considerably (Figure 4). The laser polishing process allows a decrease of $78 \%$ and $79 \%$ LMD Ti6Al4V and 316L surfaces roughness. The final $S_{a}$ is $6.01 \mu \mathrm{m}$ for the laser polished Ti6Al4V surface and $5.02 \mu \mathrm{m}$ for the $316 \mathrm{~L}$ surface. The surfaces roughness values were calculated with a $8000 \mu \mathrm{m}$ cut off filter which integrates waviness and roughness topography components without the form. This cut off enables to analyse the global evolution of the surface before and after laser polishing process. To dissociate waviness and roughness topography components cut off filters of $800 \mu \mathrm{m}$ and $250 \mu \mathrm{m}$ were used. The $250 \mu \mathrm{m} \lambda \mathrm{c}$ enables to keep the micro texture induced by the laser polishing process, such as the gap between two laser paths and the melted material micro texture. The $\lambda \mathrm{c}$ of $800 \mu \mathrm{m}$ aids to keep the smoothed waviness of the initial topography. These cut off filters show clearly the presence of waviness after laser polishing process (Figure 5 and 6 ) that can impact on the surface functionality. Both Ti6Al4V and 316L polished surfaces are composed by waviness and roughness and can be characterized through these filters values and this decomposition method. $316 \mathrm{~L}$ laser polished surface is composed by silicon oxides components. 


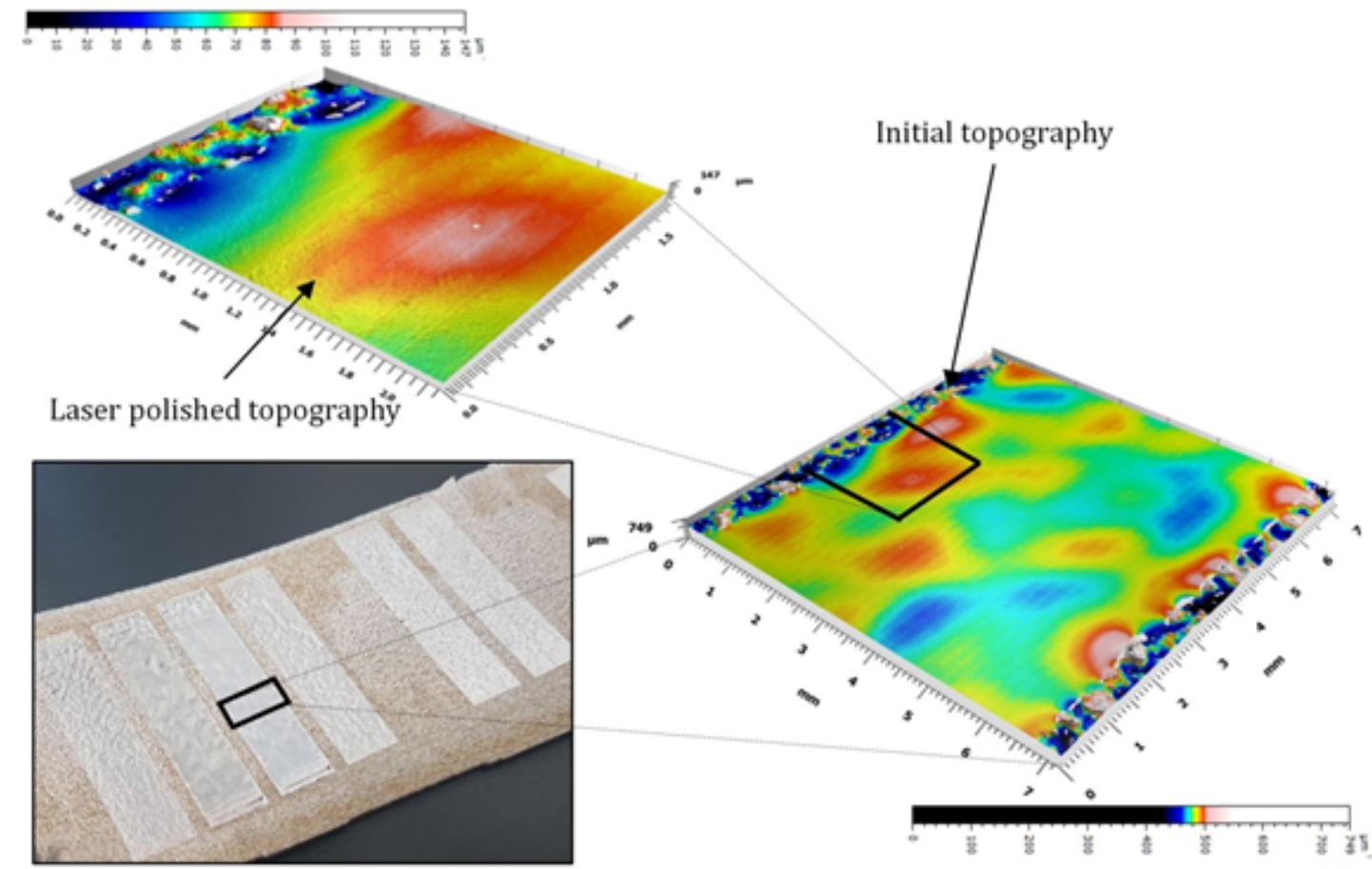

Figure 4: Ti6Al4V LMD laser polished surface

(Initial LMD surface roughness $S_{a}=27.6 \mu \mathrm{m}$; final laser polished surface roughness $S_{a}=6.01 \mu \mathrm{m}$ )

The pass number parameter impact on the final surface and enables smoothness of the surface. The increase of pass number parameter (1 and 5) allow decreasing Ti6Al4V $S_{a}(\mathrm{Cut}$ off $=800 \mu \mathrm{m})$ from $7.59 \mu \mathrm{m}$ to $5.75 \mu \mathrm{m}$.In addition, with a $250 \mu \mathrm{m}$ cut off the $S_{a}$ is reduced from 0.77 to $0.32 \mu \mathrm{m}$. The increase of pass number allows a decrease of waviness by $24 \%$ and roughness by $58 \%$ respectively. Moreover, the increase of pass number tends to increase $W s m$ by $53 \%\left(1 \mathrm{~mm}\right.$ for $1^{\text {st }}$ pass and $1.86 \mathrm{~mm}$ for 5 passes of laser polishing) regarding the waviness. As a result the pass number impact strongly on the spatial frequency of the waviness and on the roughness (Figure 7).

The increase of pass number enables to decrease the amplitude of the surface and increase the spatial frequency. The increase of the spatial frequency tends to transform waviness component into form component. Thus, the laser polishing process and pass number parameter impact on the form of the surface.

5 passes strategy helps to improve the final surface roughness. Globally, the increase of pass number, decreases waviness and roughness but increase the form component. Besides, these results highlight a limitation of laser polishing regarding the smoothing of the LMD surfaces. 


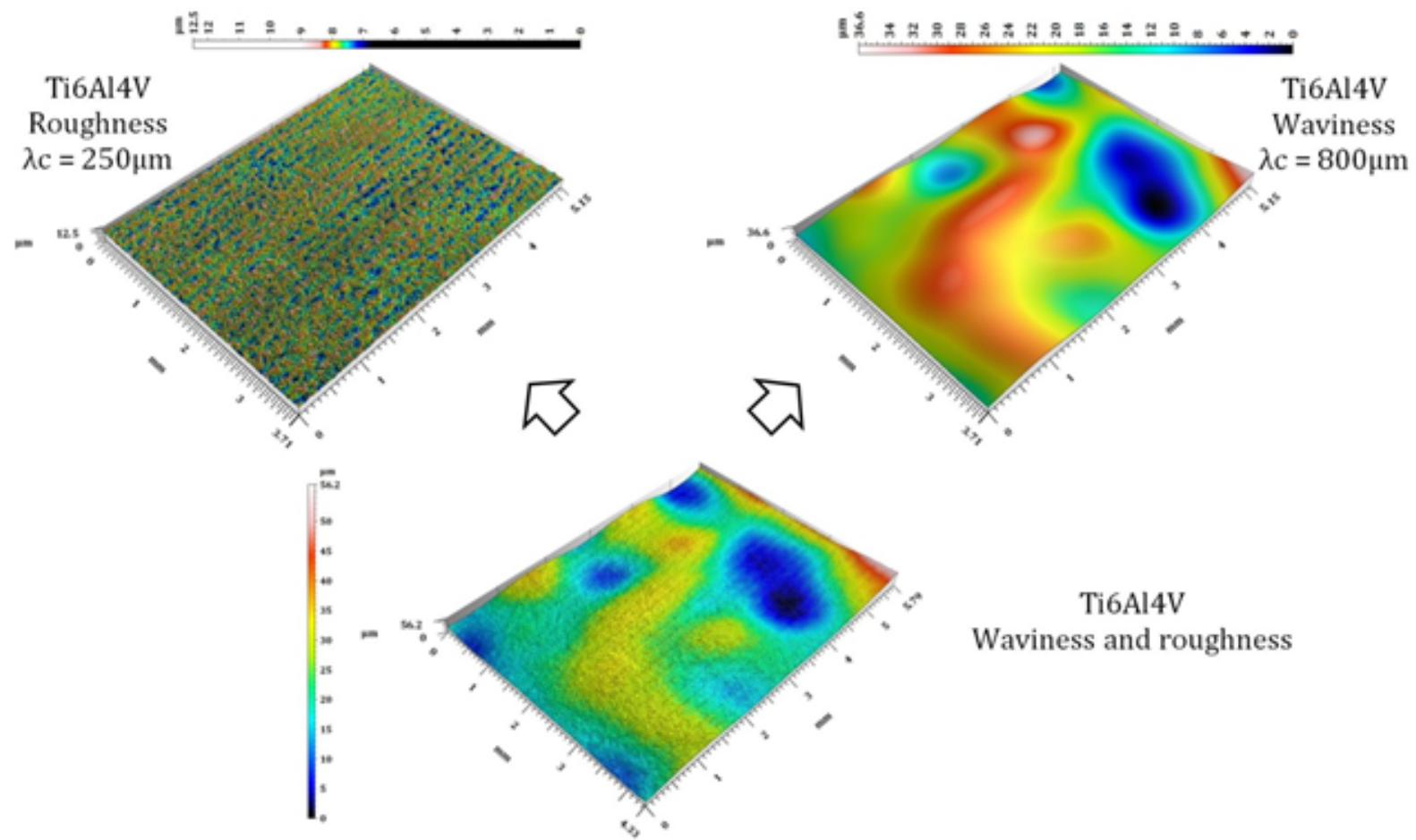

Figure 5: multi-scale decomposition of laser polished Ti6Al4V surface

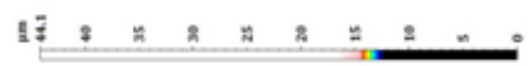

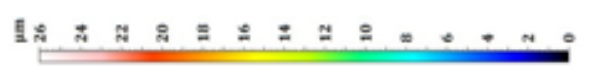

$316 \mathrm{~L}$

Roughness

$\lambda \mathrm{c}=250 \mu \mathrm{m}$
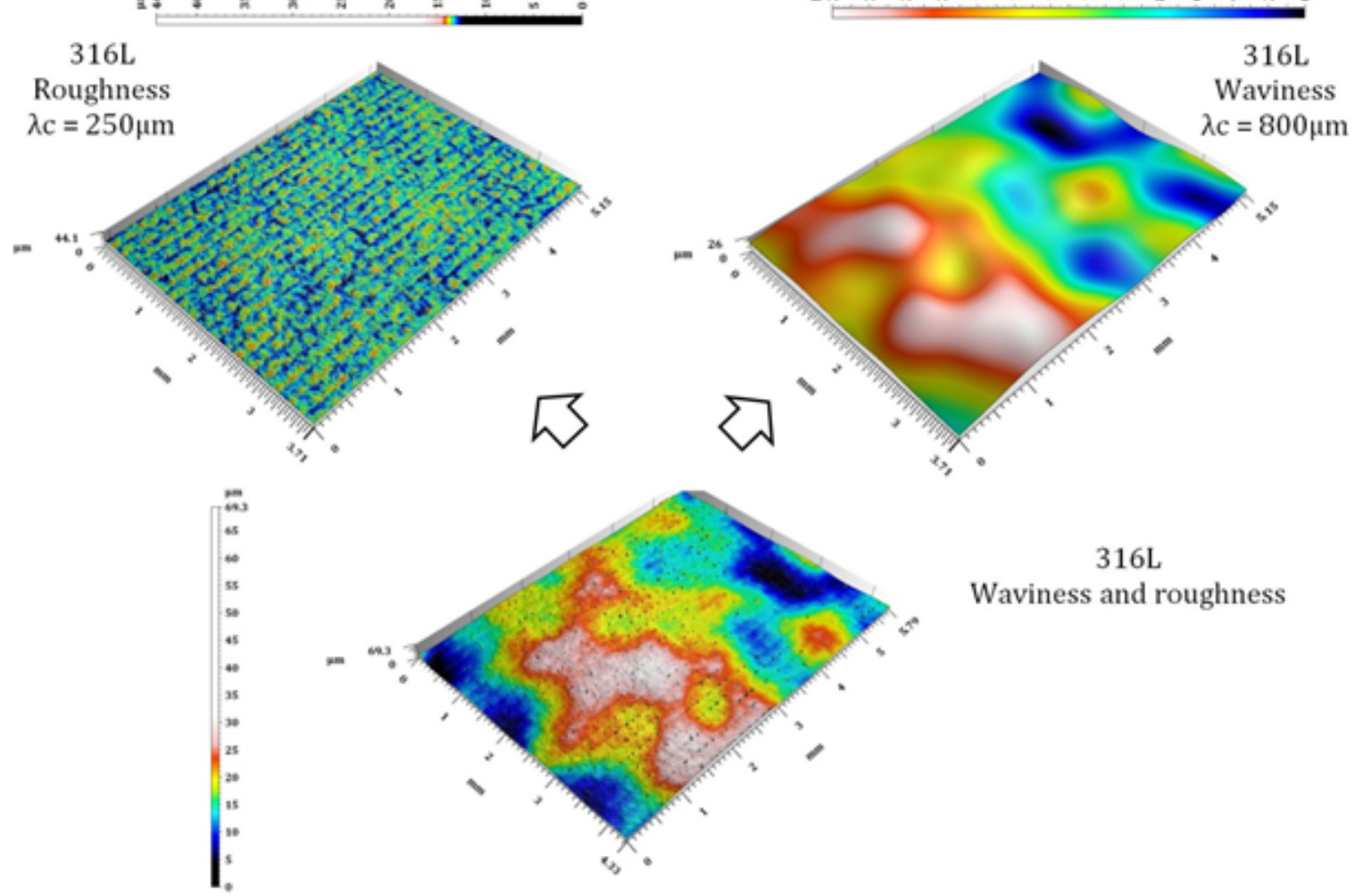

$316 \mathrm{~L}$

Waviness and roughness

Figure 6: multi-scale decomposition of laser polished 316L surface 


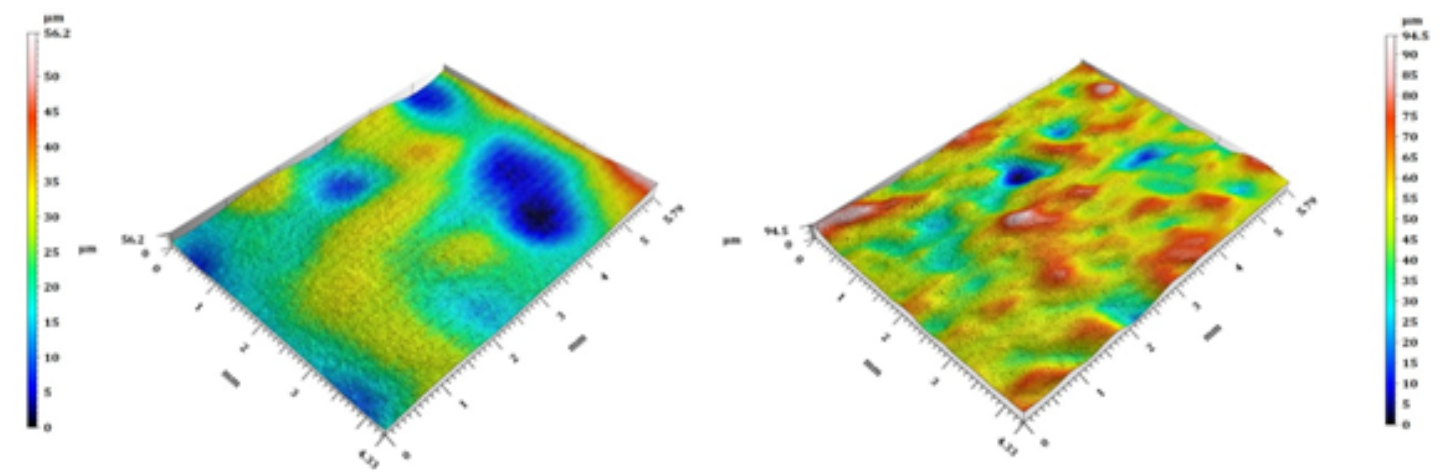

Figure 7: impact of pass number on final topography

Textures aspect ratio and autocorrelation lobe enable to analyse the texture direction and its evolution. Texture aspect ratio parameter show a texture difference for both LMD 316L and Ti6Al4V surfaces. The 316L LMD surface is isotopic, while Ti6Al4V LMD is anisotropic. This is quantified by Str parameters which are 0.01 and 0.77 respectively, this difference is caused by the waviness. After laser polishing, the texture direction had no significant difference (Figure 8). This phenomenon is directly caused by the presence of the initial topography after laser polishing. In other words, the initial topography waviness is not totally eliminated. Although, these results highlight a modification of the texture direction after laser polishing.

Besides, through 2D and 3D images analysis, no micro cracks are present after laser polishing process (Figure 9). The image and topography analysis highlight material texture along laser path but no micro cracks.

This texture is directly induced by the laser polishing process. The directional texture is caused by the gap between two laser paths and the distance between two lines is the overlap parameter value. The chaotic texture is caused by the melted material during the laser polishing process.
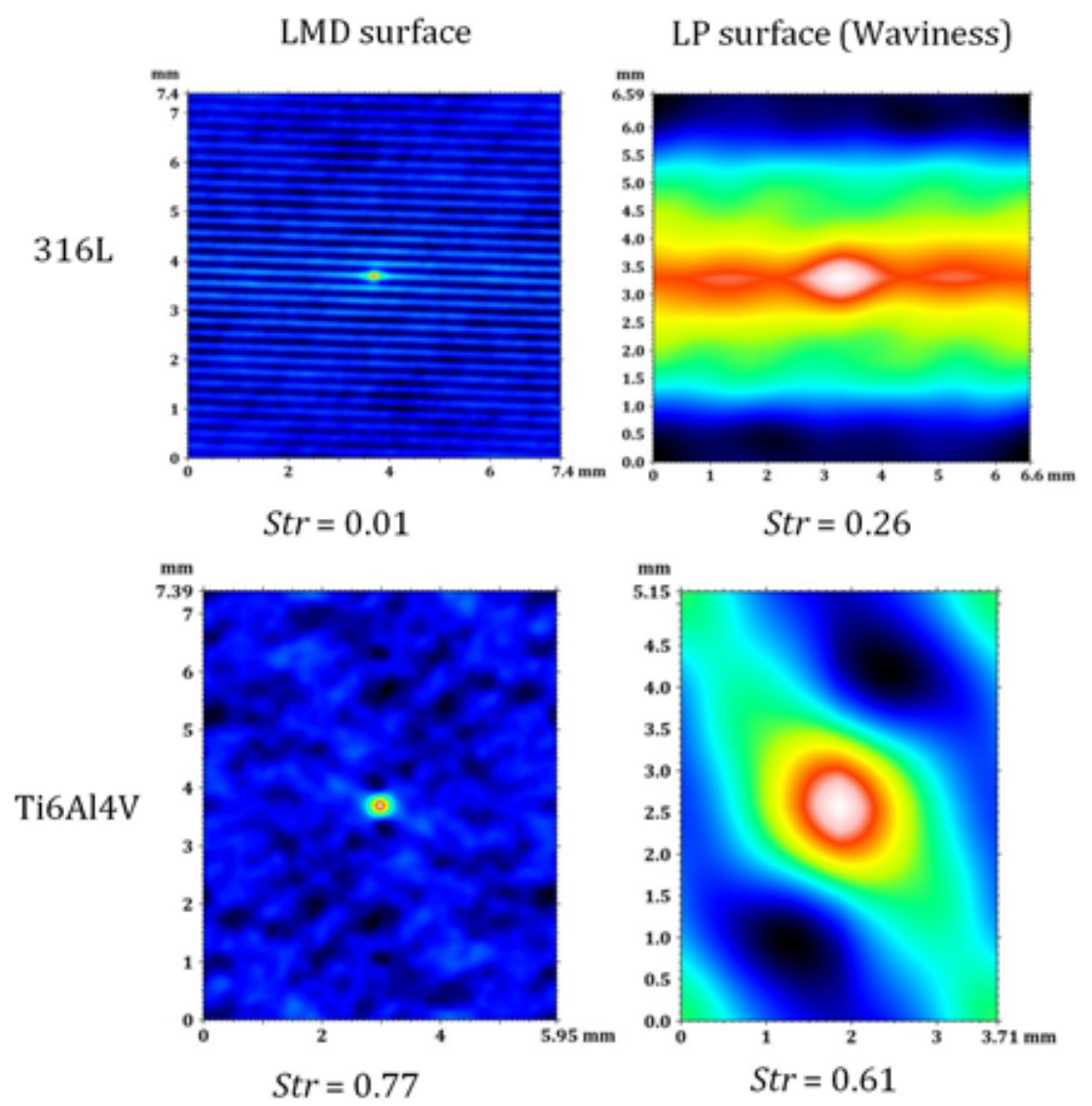

Figure 8: autocorrelation lobe results and Str parameters of LMD and laser polished surfaces

(5 passes) 


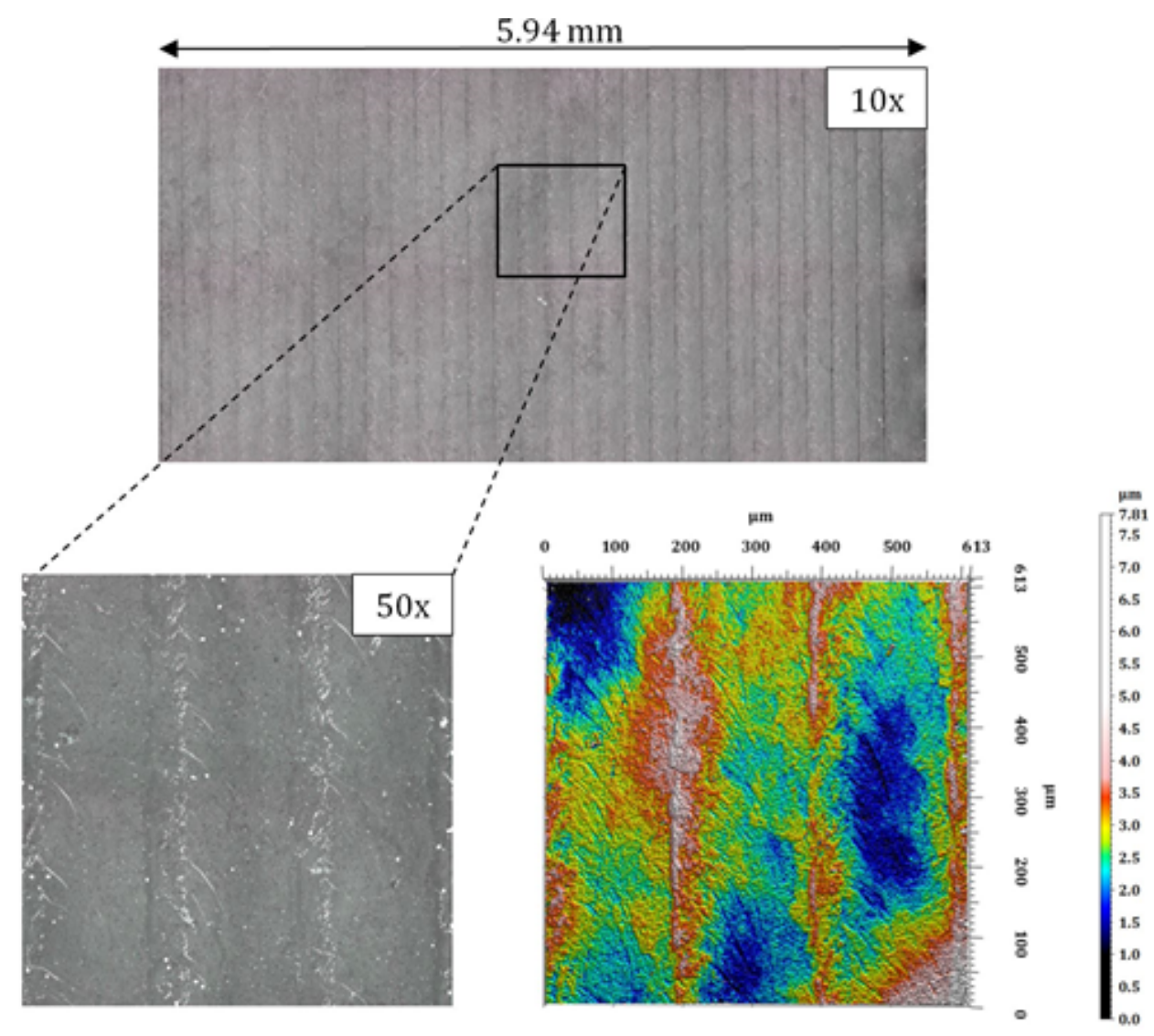

Figure 9: micro cracks analysis of laser polished surface (5 passes)

\section{Conclusion}

This study analyse laser polishing of Ti6Al4V LMD surfaces. In order to analyse the impact of laser polishing process, the Ti6Al4V surfaces are compared to $316 \mathrm{~L}$ Surfaces. The laser polishing process allows decrease in the LMD Ti6Al4V surface roughness (Cut off filter $=8000 \mu \mathrm{m}$ ) by $78 \%$. The initial $S_{a}$ is $27.6 \mu \mathrm{m}$ and the final $6.01 \mu \mathrm{m}$. This surface improvement highlights the efficiency of laser polishing process. $8000 \mu \mathrm{m}$ cut off filter enables to analyse the global evolution of the surfaces while 250 and $800 \mu \mathrm{m}$ enables to analyse the local evolution.

The laser polishing process is impact on both waviness and roughness of the topography highlighted by 250 and $800 \mu \mathrm{m}$ of cut off filters. The pass number parameter impacts on roughness, increases the spatial frequency of the surface waviness and tends to transfer waviness into form component. Globally, the increase of pass number allows improving the surface smoothing.

This study highlights the importance to analyse the surfaces with a multi-scales approach and with amplitude $\left(S_{a}\right)$, spatial (Wsm) and texture (Str and autocorrelation lobe) parameters. Through the texture aspect ratio, and the autocorrelation lobe the presented results highlight the impact of the initial topography after laser polishing. The final topography is not totally eliminated after laser finishing process which shows the limitation of laser polishing process. The operating parameters of laser polishing enable to smooth initial LMD Ti6A14V surfaces without micro cracks.

\section{Perspectives}

Further researches will focuses on the optimisation of the initial topography regrading laser polishing process. Moreover, more material analysis will be performed to measure the mechanical properties of both LMD and laser polished surfaces. Finally, simulation of laser polished LMD surfaces will be investigated.

\section{6. $\underline{\text { References }}$}

[1]: E. C. Hussam, B. Courant, S. Branchu, JY Hascoët, R. Guillén, 2012, "Direct Laser Fabrication process with coaxial powder projection of $316 \mathrm{~L}$ steel. Geometrical characteristics and microstructure characterization of wall structures" Journal Optics and Lasers in Engineering, Vol 50-12, pp 1779-1784. 
[2]: Tugdual Amaury Le Néel, Jean-Yves Hascoët, 2018, "A review on additive manufacturing of sand molds by binder jetting and selective laser sintering", Rapid Prototyping Journal, Vol. 24 Issue: 8, pp.1325-1336.

[3]: Grant Marchelli, Renuka Prabhakar, Duane Storti, Mark Ganter, 2011, "The guide to glass 3D printing: developments, methods, diagnostics and results", Rapid Prototyping Journal, Vol. 17 Issue: 3, pp.187-194.

[4]: J. Deckers, J. Vleugels, J.-P. Kruth, 2014,”Additive manufacturing of ceramics: a review”, Journal of ceramic science and technology, 05 [04], 245-260.

[5]: T. Hofstätter, D. B. Pedersen, G. Tosello and H. N. Hansen, 2017, “Applications of fiber-reinforced polymers in additive manufacturing" Procedia CIRP 66, 312-316.

[6]: J. Gardan and L. Roucoules, 2014, “3D printing device for numerical control machine and wood deposition” International Journal of Engineering Research and Applications, pp. 123-131.

[7]: C. Gosselin, R. Duballet, Ph. Roux, N. Gaudillière, J.Dirrenberger, Ph. Morel, 2016, "Large-scale 3D printing of ultra-high performance concrete a new processing route for architects and builders", Material and Design 100, 102 - 109.

[8]: Jie Sun, Zhuo Peng, Weibiao Zhou, Jerry Y.H.Fuh, Geok Soon Hong, Annette Chiu, 2015, “A Review on 3D Printing for Customized Food Fabrication" Procedia Manufacturing, Volume 1, Pages 308-319.

[9]: Benoit Rosa, Perrine de Villemagne, Franck Halary, Jerome Guicheux, Catherine Le Visage, Jean Yves Hascoët, “4Dbioprinted functionally graded material”, Bioregate Forum 2018, December, Nantes.

[10]: Jean-Yves Hascoët, Benoit Rosa, Perrine de Villemagne, Franck Halary, "Modeling of 4D-bioprinting process for improved final resolution of a tissue", Workshop "3D Bioprinting in Cancer Research, July 5th 2017, Nantes.

[14]: P Muller, JY Hascoet, P Mognol, 2014, “ Toolpaths for additive manufacturing of functionally graded materials (FGM) parts”, Rapid Prototyping Journal 20 (6), 511-522.

[15]: Benoit Rosa, Antoine Brient, Serge Samper and Jean-Yves Hascoët, 2016, "Influence of additive laser manufacturing parameters on surface using density of partially melted particles”, Surf. Topogr.: Metrol. Prop. 4, 045002, 2016.

[16]: Qinghua Wang, Justin D. Morrow, Chao Ma, Neil A. Duffie, Frank E. Pfefferkorn, Surface prediction model for thermocapillary regime pulsed laser micro polishing of metals, Journal of Manufacturing Processes, Volume 20, Part 1, Pages 340348, 2015.

[17]: L. Giorleo, E. Ceretti, C. Giardini, Ti surface laser polishing: effect of laser path and assist gas, 9th CIRP Conference on Intelligent Computation in Manufacturing Engineering - CIRP ICME '14, Procedia CIRP 33, 446 - 451, 2015.

[18]: C.P. Ma, Y.C. Guan, W. Zhou, "Laser polishing of additive manufactured Ti alloys", Optics and Lasers in Engineering, Volume 93, Pages 171-177, 2017.

[19]: Yingtao Tian, Wojciech S. Gora, Aldara Pan Cabo, Lakshmi L. Parimi, Duncan P. Hand, Samuel Tammas-Williams, Philip B. Prangnell, "Material interactions in laser polishing powder bed additive manufactured Ti6Al4V components", Additive Manufacturing, Volume 20, Pages 11-22, 2018.

[20]: Yu-Hang Li, Bing Wang, Cheng-Peng Ma, Zhi-Hao Fang, Long-Fei Chen, Ying-Chun Guan and Shou-Feng Yang, "Material Characterization, Thermal Analysis, and Mechanical Performance of a Laser-Polished Ti Alloy Prepared by Selective Laser Melting”, Metals, 9, 112, 2019.

[21]: Swee Leong Sing, Florencia Edith Wiria, Wai Yee Yeong, "Selective laser melting of titanium alloy with $50 \mathrm{wt} \%$ tantalum: Effect of laser process parameters on part quality", International Journal of Refractory Metals and Hard Materials, Volume 77, Pages 120-127, 2018.

[22]: B. Rosa, J.Y. Hascoët, P. Mognol, “Laser polishing of additive laser manufacturing surfaces”, Journal of Laser Application, Vol. 27, Number S2, 2015.

[23]: B. Rosa, J.Y. Hascoët, P. Mognol, "Modelling and optimization of laser polishing of additive laser manufacturing surfaces", Rapid Prototyping Journal, Volume 22, Number 6, 956-964, 2016.

[24]: Standard ISO 4288, Geometrical Product Specifications (GPS). Surface texture: Profile method, rules and procedures for the assessment of surface texture, 1998. 